\title{
Optical properties of Swift long gamma-ray bursts
}

\section{Andrea Melandri*}

INAF - Osservatorio Astronomico Brera, Via E. Bianchi 46, I-23807, Merate (LC), Italy

E-mail: andrea.melandriebrera.inaf.it

In the era of rapid follow-up, well-sampled optical light curves (covering a wide range in time, brightness and redshift) provide a powerful probe of the physics of gamma-ray bursts, their ejecta and their environments. Here we present a comprehensive study of optical properties of the complete BAT6 sample ${ }^{\dagger}$ of Swift bright and long gamma-ray bursts. In the optical band we investigated their light curves behaviour, luminosity, rest-frame extinctions and intrinsic darkness. We also searched for any possible correlations between high-energy and optical properties. Finally, we summarise the main results of the multi-wavelengths analysis of the complete BAT6 sample.

Swift: 10 Years of Discovery,

2-5 December 2014

La Sapienza University, Rome, Italy

\footnotetext{
*Speaker.

${ }^{\dagger}$ Dedicated web page: www.brera.inaf.it/utenti/melandri/BAT6_Sample
} 


\section{Introduction}

The large number of events detected by the Swift satellite [1] in its first 6.5 years of life, has allowed the creation of an uniform, unbiased and complete sample of long and bright gamma-ray bursts (LGRBs), the so-called BAT6 sample [2]. The sample consists of 58 events selected to be relatively bright in the 15-150 keV Swift Burst Alert Telescope (BAT) band, detected between Nov 2004 and May 2011, and with favourable conditions for ground-based multi-wavelength follow-up observations. Having the highest level of completeness in redshift $(\sim 95 \%)$, the exploitation of this complete LGRBs sample allowed a series of studies of the global properties of this class of event at all wavelengths. In this paper we summarise the main findings of the BAT6 sample, with a particular focus on the statistical results obtained in the optical domain. For a more exhaustive and detailed explanation of each result we refer the reader to single focused paper that we published on each topic.

\section{Optical LGRBs light curves}

We were able to compute the rest-frame light curves in the X-ray and optical bands for 55 and 47 objects, respectively. After taking into account for any possible source of absorption (Galactic and intrinsic) that could affect the observed fluxes, we computed the X-ray and optical luminosities and compared the behaviour in these two bands [3]. For the majority of the events $(\sim 70 \%)$, the rest-frame light curves in the X-ray and optical bands have a different evolution, indicating distinct emitting regions and/or mechanisms. While the X-ray emission seems to still have a strong contribution from the prompt emission or from some late time central engine activity, the emission in the optical band is mostly (if not completely) dominated by the afterglow emission.

Moreover, the optical luminosity of LGRBs, estimated in the $R$-band at a rest-frame time $t_{\mathrm{RF}}=12 \mathrm{hr}$, has a uniform distribution (Fig. 1, left panel) around a mean value $\log \left(\mathrm{L}_{\mathrm{R}}\right)=29.9$ (dispersion $\sigma=0.8$ ). In fact, we unarguably demonstrated that no bimodality is present, as found in previous published studies based on incomplete samples.

\section{Optical extinction}

Using optical and X-ray information, the rest-frame extinction (or limits on it) could be estimated for the $91 \%$ of the cases (53 events out of 58). The distribution of the rest-frame extinction seems to be bimodal, with the majority of the events clustered at virtually zero extinction and several events at higher extinction (Fig. 1, right panel). In particular, 87\% of LGRBs have an extinction smaller than about $2 \mathrm{mag}$ ( $\sim 50 \%$ smaller than 0.3-0.4 mag), while the remaining $13 \%$ of events are suffering from high extinction (more than $2 \mathrm{mag}$, see [4]). The percentage of events with large extinction could be slightly higher if we assume that the events without redshift measurement in our sample are likely part of this class. Therefore, this fraction is well in the range of the estimated percentage of dark bursts (see next Section).

There is no clear redshift dependence of the derived rest-frame extinction up to $z \sim 4$. However, we find that the largest extinction is detected in the redshift range $1.5<z<2$, close to the expected peak of the star formation rate [4]. 

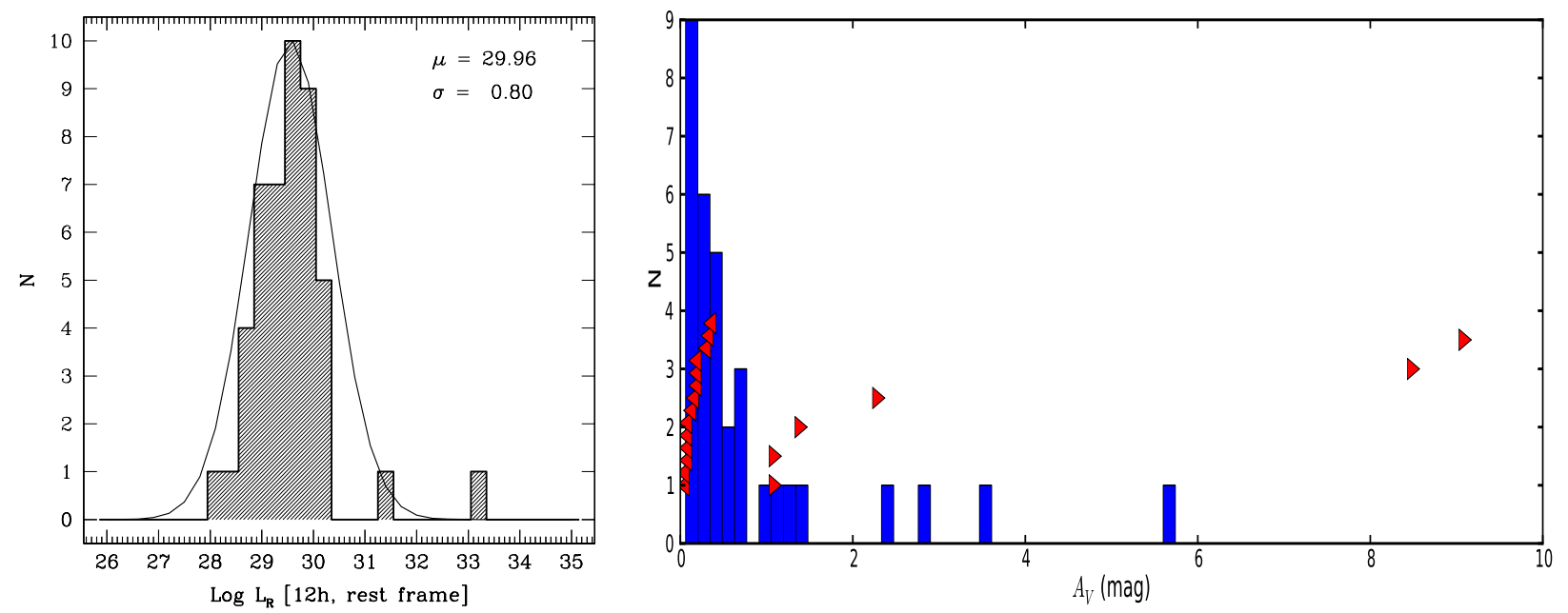

Figure 1: Left: optical rest-frame luminosity of LGRBs ( $R$-band, $\mathrm{erg} \mathrm{s}^{-1} \mathrm{~Hz}^{-1}$ ) distribution at $t_{\mathrm{RF}}=12$ hr. The mean value of the distribution is $\mu=29.9$ with a dispersion $\sigma=0.8$ [3]. Right: histogram of the computed rest-frame $A_{V}$ for the LGRBs. Upper and lower limits are also reported (red triangles) [4].

\section{Dark GRBs}

We studied in details the properties of the subclass of optically dark bursts in the BAT6 sample [5]. According to the definition of dark burst from [6], we find that:

1. there is a genuine population ( $\sim 25-35 \%)$ of optically obscured events (Fig. 2, left panel);

2. the majority of those are dark from very early time, meaning that their darkness is likely due to the Ly $\alpha$ absorption in the optical bands;

3. dark bursts do not have different prompt properties compared to the normal events. At the same time they are not only fainter in the optical but also brighter in the X-ray;

4. the higher X-ray column densities observed for the dark bursts in our sample (Fig. 2, right panel) clearly indicate that they formed in metal-rich environments where a fair amount of dust must be present;

5. the redshift distribution of the dark bursts population is the same as the whole sample.

All that considered, dark bursts can be hardly explained in the context of the high-z scenario or the low-density scenario. The most plausible explanation is left to be found in the context of the dust scenario and in fact high values of the X-ray column densities are hint of high local absorption.

\section{Host galaxies}

We started to investigate also the properties of the host galaxies for LGRBs in our sample. From the spectral energy distribution analysis of the host galaxies at $z<1$ of the BAT6 sample (14 events) we were able to determine their stellar masses with good accuracy.

We find that LGRBs hosts at $z<1$ have lower luminosities and stellar masses than expected if LGRBs were unbiased star formation tracers. The preference for low metallicities environment $(\mathrm{Z}$ 

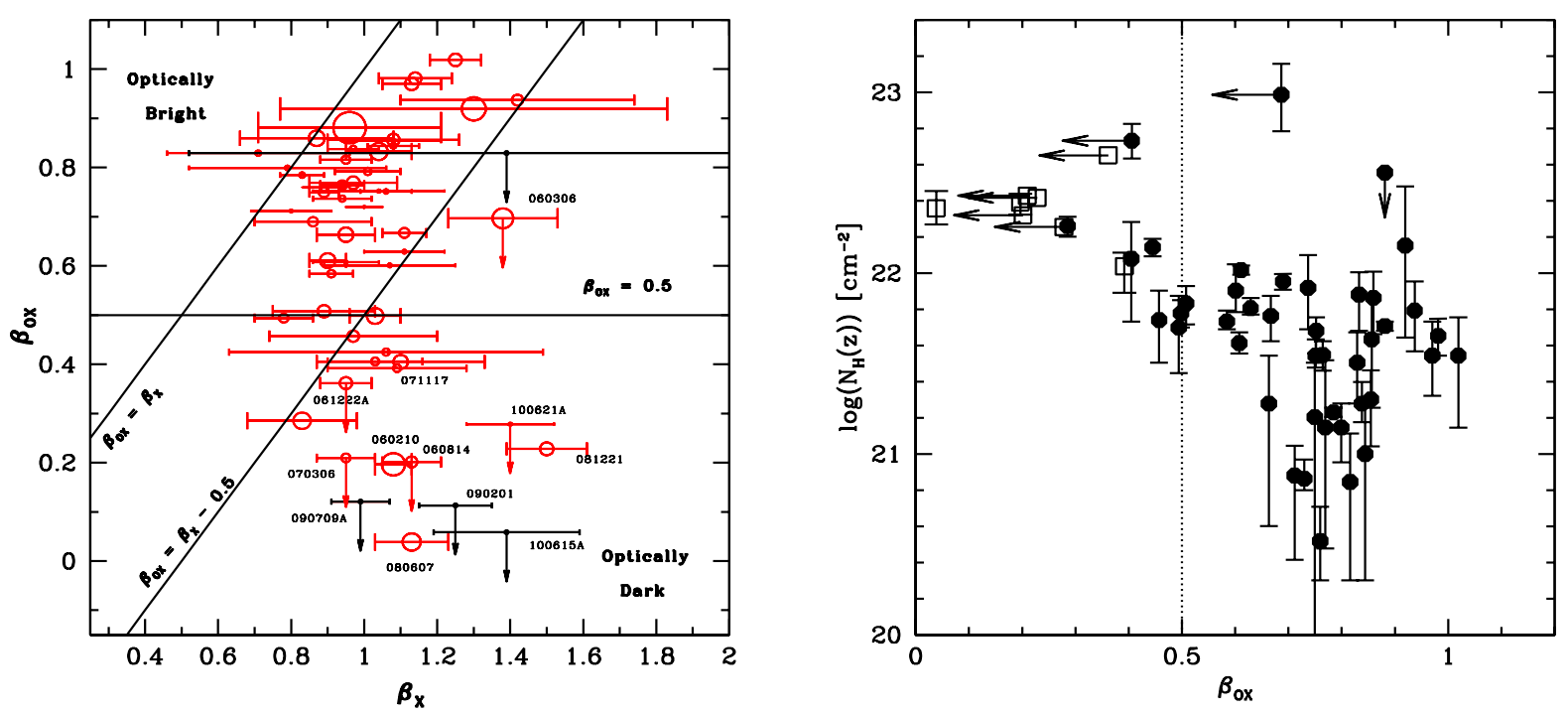

Figure 2: Left: dark bursts distribution in the BAT6 sample. The dimension of the symbol is a direct visual of the value of the redshift of the GRB; the larger the symbol the bigger the associated redshift [5]. Right: column density $\left(\mathrm{N}_{\mathrm{H}}\right)$ as a function of the spectral index $\beta_{\mathrm{OX}}$ (from [11]). Open squares (filled circles) indicate dark (non-dark) GRBs according to [6]. Limits on these two parameters are indicated with arrows.

$<0.5 Z_{\odot}$ ) inferred by the comparison with the simulations (Fig. 3, left panel) can be a consequence of the particular conditions needed for the progenitor star to produce a GRB [7].

\section{Other BAT6 results}

- The first result obtained with the BAT6 sample is that in order to account for the observed redshift distribution of LGRBs a strong evolution in luminosity $\left(\delta_{l}=2.3 \pm 0.6\right)$ or in density $\left(\delta_{d}=1.7 \pm 0.5\right)$ is required, on top of the known cosmic evolution of the SFR [2]. These two scenarios are consistent with each other and this calls for other indicators in order to distinguish among different evolutionary models (Fig. 3, right panel). On the basis of our models we predicted that $3 \%-5 \%$ of the bursts detected by Swift lie at redshift z $>5$. Subsequently, this prediction strengthened the result found by [5] that high-z bursts contributed only marginally to the observed fraction $(\sim 30 \%)$ of dark bursts.

- Using the complete sample we were able to study the spectral-energy correlations in an unbiased way. The slopes of the well-known GRBs correlations defined by the BAT6 sample are 0.61 (for the $\mathrm{E}_{\text {peak }}-\mathrm{E}_{\text {iso }}$ correlation) and 0.53 (for the $\mathrm{E}_{\text {peak }}-\mathrm{L}_{\text {iso }}$ correlation). The estimated slopes are consistent with the values found with larger but incomplete samples. This means that negligible selection effects for spectral-energy correlations are present. Moreover, we find no evolution with redshift of these correlations. See [8] and [9] for details.

- The mean value of the total distribution of intrinsic column densities $\left(\mathrm{N}_{\mathrm{H}}\right)$ of bright LGRBs $(21.7 \pm 0.5)$ is consistent with the mean value of previous incomplete samples (i.e. $21.9 \pm$ 0.1 , from [10]). The value of $\mathrm{N}_{\mathrm{H}}$ increases with redshift, with some contribution due to the 

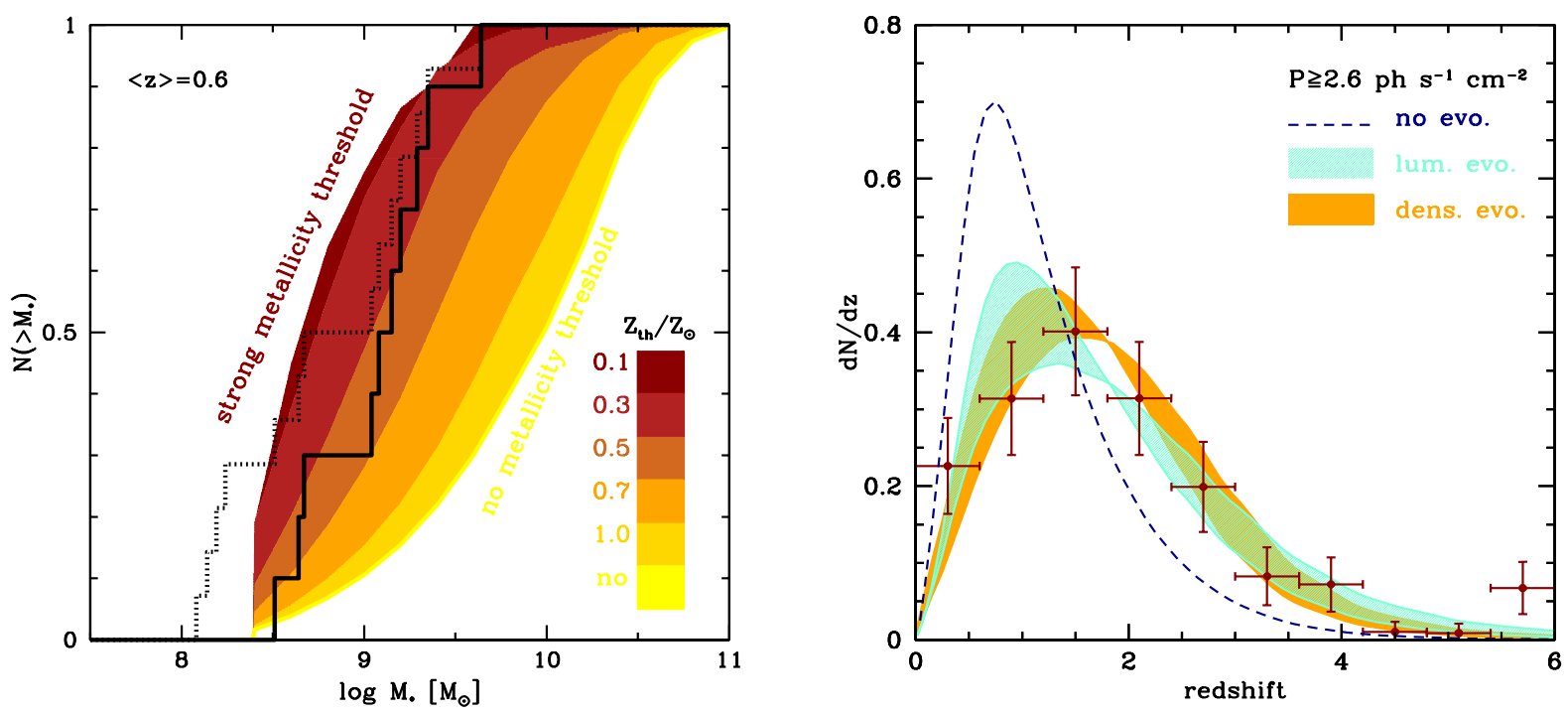

Figure 3: Left: Cumulative distribution of the stellar masses of the LGRB hosts in the BAT6 sample (dotted line: whole sample; solid line: with $\log M_{\star}>8.4 \mathrm{M}_{\odot}$ compared to that obtained by LGRB host galaxy simulations [7]. The observed distribution is explained by a metallicity cut favouring low metallicities ( $\mathrm{Z}<$ $\left.0.5 Z_{\odot}\right)$. Right: normalised redshift distribution of LGRBs. The observed redshift distribution (red points) are compared with the "no-evolution" case (blue dashed line) and with models for luminosity (light blue) and density (orange) evolution [2].

intervening systems along the line of sight. There is a strong correlation between LGRBs darkness and $\mathrm{N}_{\mathrm{H}}$, supporting the picture in which the darkness of a GRB is in most cases due to absorption by circumburst material [11].

- A significant correlation was found between the rest-frame X-ray luminosity $\left(\mathrm{L}_{\mathrm{X}}\right)$ with the prompt emission isotropic energy $\left(\mathrm{E}_{\text {iso }}\right)$ and the isotropic peak luminosity $\left(\mathrm{L}_{\mathrm{iso}}\right)$. These correlations are stronger at early times ( $t_{\mathrm{RF}}=5$ minutes) while theirs significance decrease with time. This strongly indicates that the $\mathrm{L}_{\mathrm{X}}$ is still affected at early times by the contribution of the prompt emission, while at late times the most significant contribution comes from the afterglow emission [12].

- The majority of the BAT6 LGRBs have been observed at radio frequencies ( $\sim 70 \%)$. Assuming some standard afterglow parameters (see [13] for full details) we derived the radio flux distributions of all the GRBs that can be detected from Earth. We estimated also the number

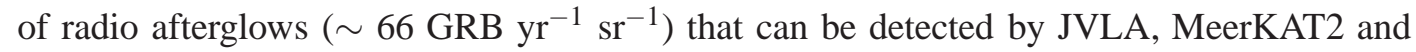
SKA [14]. These radio detection rate depend on the exposure times, and on the observing frequency and time. The sooner the radio observation (between 1-10 days post-burst) the higher the rate of radio afterglows detection.

- Within our sample we identified 10 events that show a precursor in the Swift/BAT 15-150 $\mathrm{keV}$ energy band. These events can be explained assuming that the central GRB engine is a newly born magnetar and the precursor and the prompt emission arises from the accretion 
of matter onto its surface [15]. The fraction of GRBs powered by a magnetar is even higher if we interpret the plateau in the X-ray light curves of GRB afterglows as due to continuous energy injection from the magnetar spin-down power, resulting in at least $\sim 80 \%$ of the entire population.

- We applied the standard procedure to find out the GRB time-integrated spectral lag to the LGRBs of the BAT6 sample. For 50 events it was possible to determine the value of the spectral lag, and we do not find evidence for any lag-luminosity correlation. Our result suggests that the previously known lag-luminosity correlation is actually a boundary on that plane [16].

\section{Conclusions}

The exploitation of the complete BAT6 sample has allowed a better understanding of the properties of LGRBs at different wavelengths. A series of studies have been published to report the focused analysis of each single aspect. These results, due to the high level of completeness of the starting sample, can be considered as representative of the whole class of LGRBs. Therefore, future observations from the ground, aimed to maintain the completeness of such a sample as high as possible, are extremely important.

At present time the complete BAT6 sample has reached $\sim 100$ events. A dedicated webpage has been created (www.brera.inaf.it/utenti/melandri/BAT6_Sample), that will be updated with future results of the extended BAT6 sample.

\section{References}

[1] N., Gehrels, G., Chincarini, P., Giommi, et al., The Swift gamma-ray burst Mission, ApJ, 611 (2004) 1005

[2] R., Salvaterra, S., Campana, S. D., Vergani, et al., A Complete Sample of Bright Swift Long gamma-ray bursts. I. Sample Presentation, Luminosity Function and Evolution, ApJ, 749 (2012) 68

[3] A., Melandri, S., Covino, D., Rogantini, et al. Optical and X-ray rest-frame light curves of the BAT6 sample, A\&A, 565 (2014) 72

[4] S., Covino, A., Melandri, R., Salvaterra, et al., Dust extinctions for an unbiased sample of gamma-ray burst afterglows, MNRAS, 432 (2013) 1231

[5] A., Melandri, B., Sbarufatti, P., D’Avanzo, et al., The dark bursts population in a complete sample of bright Swift long gamma-ray bursts, MNRAS, 421 (2012) 1265

[6] A. J., van der Horst, C., Kouveliotou, N., Gehrels, et al. Optical Classification of gamma-ray bursts in the Swift Era, ApJ, 699 (2009) 1087

[7] S. D., Vergani, R., Salvaterra, J., Japelj, et al. Are LGRBs biased tracers of star formation? Clues from the host galaxies of the BAT6 complete sample of LGRBs. I: Stellar mass at $z<1, A \& A$ submitted (arXiv:1409.7064)

[8] L., Nava, R., Salvaterra, G., Ghirlanda, et al., A complete sample of bright Swift long gamma-ray bursts: testing the spectral-energy correlations, MNRAS, 421 (2012) 1256 
[9] G., Ghirlanda, G., Ghisellini, L., Nava, et al., The impact of selection biases on the $E_{\text {peak }}-L_{\mathrm{ISO}}$ correlation of gamma-ray bursts, MNRAS, 422 (2012) 2553

[10] S., Campana, C. C., Thöne, A., de Ugarte Postigo, et al. The X-ray absorbing column densities of Swift gamma-ray bursts, MNRAS, 402 (2010) 2429

[11] S., Campana, R., Salvaterra, A., Melandri, et al., The X-ray absorbing column density of a complete sample of bright Swift gamma-ray bursts, MNRAS, 421 (2012) 1697

[12] P., D’Avanzo, R., Salvaterra, B., Sbarufatti, et al. A complete sample of bright Swift gamma-ray bursts: X-ray afterglow luminosity and its correlation with the prompt emission, MNRAS, 425 (2012) 506

[13] G., Ghirlanda, G., Ghisellini, R., Salvaterra, et al. The faster the narrower: characteristic bulk velocities and jet opening angles of gamma-ray bursts, MNRAS, 428 (2013) 1410

[14] G., Ghirlanda, R., Salvaterra, D., Burlon, et al. Radio afterglows of a complete sample of bright Swift GRBs: predictions from present days to the SKA era, MNRAS, 435 (2013) 2543

[15] M. G., Bernardini, S., Campana, G., Ghisellini, et al. How to Switch a gamma-ray burst On and Off through a Magnetar, ApJ, 775 (2013) 67

[16] M. G., Bernardini, G., Ghirlanda, S., Campana, et al. Comparing the spectral lag of short and long gamma-ray bursts and its relation with the luminosity, MNRAS, 446 (2015) 1129 